\title{
Caracterização de Aeromonas spp isoladas de neonatos hospitalizados
}

\author{
Characterization of Aeromonas spp \\ isolates from newborns hospitalized
}

\author{
Christiane Soares Pereira ${ }^{1}$, Simone Duarte Amorim ${ }^{1}$, André Felipe das Mercês Santos ${ }^{1}$, \\ Cristhiane Moura Falavina dos Reis ${ }^{2}$, Grace Nazareth Diogo Theophilo ${ }^{1}$ \\ e Dália dos Prazeres Rodrigues ${ }^{1}$
}

\begin{abstract}
RESUMO
Aeromonas spp é reconhecida como patogênica para o homem após o consumo de água e alimentos contaminados. Na presente investigação, foram avaliadas 2.323 amostras de swabs retais de neonatos hospitalizados no Rio de Janeiro objetivando o isolamento de Aeromonas. As amostras foram coletadas e enviadas ao Laboratório de Referência Nacional de Cólera e outras enteroinfecções bacterianas, Instituto Oswaldo Cruz, Fundação Oswaldo Cruz. Os swabs foram submetidos ao enriquecimento em água peptonada alcalina adicionada de 1\% de cloreto de sódio (NaCl) e água peptonada alcalina adicionada de $3 \%$ de $\mathrm{NaCl}\left(37^{\circ} \mathrm{C} / 18-24 \mathrm{~h}\right)$ e semeadas em agar seletivo para Pseudomonas aeromonas (Agar GSP). Foram isoladas 56 cepas de Aeromonas assim distribuídas: Aeromonas caviae (42,8\%), Aeromonas media (25\%), Aeromonas veronii biogrupo sobria (10,7\%), Aeromonas hydrophila (9\%), Aeromonas veronii biogrupo veronii (5,3\%), Aeromonas sobria (1,8\%), Aeromonas jandaei (1,8\%), Aeromonas schubertii (1,8\%) e Aeromonas sp (1,8\%). Foi observada resistência a uma ou mais drogas antimicrobianas em $26,8 \%$ das cepas. Considerando a relevância de Aeromonas torna-se urgente alertar sobre sua importância para o controle de infecções hospitalares.
\end{abstract}

Palavras-chaves: Aeromonas spp. Neonatos. Resistência antimicrobiana. Saúde Pública.

\begin{abstract}
Aeromonas spp is recognized as pathogenic to humans after consumption of contaminated water and food. In the present investigation, 2,323 rectal swab samples from newborns hospitalized in Rio de Janeiro were evaluated with a view to isolating Aeromonas. The samples were collected and sent to the national reference laboratory for cholera and other bacterial intestinal infections, at the Oswaldo Cruz Institute of the Oswaldo Cruz Foundation. The swabs were subjected to enrichment in alkaline peptonated water with the addition of $1 \%$ sodium chloride ( $\mathrm{NaCl})$ and alkaline peptonated water plus $3 \% \mathrm{NaCl}\left(37^{\circ} \mathrm{C} / 18-24 \mathrm{~h}\right)$ and were streaked onto agar that was selective for Pseudomonas-Aeromonas (GSP Agar). Fifty-six Aeromonas strains were isolated, distributed as follows: Aeromonas caviae (42.8\%), Aeromonas media (25\%), Aeromonas veronii biogroup sobria (10.7\%), Aeromonas hydrophila (9\%), Aeromonas veronii biogroup veronii (5.3\%), Aeromonas sobria (1.8\%), Aeromonas jandaei (1.8\%), Aeromonas schubertii (1.8\%) and Aeromonas sp (1.8\%). Resistance to one or more antimicrobial drugs was observed in $26.8 \%$ of the strains. Considering the importance of Aeromonas, there is an urgent need to warn about this in relation to nosocomial infection control.
\end{abstract}

Key-words: Aeromonas spp. Newborns. Antimicrobial resistance. Public health.

Aeromonas spp compreendem bactérias Gram negativas pertencentes à família Aeromonadaceae cujo habitat é predominantemente aquático (dulcícola e marinho) e possuem ampla distribuição geográfica. São microrganismos de circulação ambiental e com capacidade de ocasionar patogenias moderadas ou graves tanto no homem quanto nos animais. Atualmente determinadas espécies de Aeromonas tem sido reconhecidas como patógenos causadores de infecções intestinais ou extra-intestinais no homem assumindo destacada relevância epidemiológica em casos de infecções oportunistas em pacientes

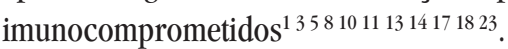

Os principais fatores de virulência destes patógenos estão associados à presença de enterotoxinas, citotoxinas, hemolisinas, aerolisinas e proteases que possuem habilidade de adesão as

1. Laboratório de Referência Nacional de Cólera e Outras Enteroinfecções Bacterianas, Instituto Oswaldo Cruz, Fundação Oswaldo Cruz, Rio de Janeiro, RJ. 2. Laboratório de Zoonoses Bacterianas, Instituto Oswaldo Cruz, Fundação Oswaldo Cruz, Rio de Janeiro, RJ.

Endereço para correspondência: Dra Christiane Soares Pereira. Laboratório de Referência Nacional de Cólera e outras Enteroinfecções Bacterianas/IOC/FIOCRUZ. Av. Brasil 4365/3 andar, Pavilhão Rocha Lima, Manguinhos, 21045-900 Rio de Janeiro, RJ.

Tel: 5521 2270-6565; ramal 320.

e-mail: chrisspm@ioc.fiocruz.br

Recebido para publicação em: 18/10/2007

Aceito em: 07/03/2008 
células teciduais causando diversos graus de injúria tecidual. A maioria dos casos de infecção intestinal no homem é representada por sintomas de diarréia aguda e a severidade da doença depende diretamente dos fatores de virulência envolvidos e do estado imunológico do paciente 2311152022 .

As principais manifestações extra-intestinais são representadas por casos de celulites, fascites necrotizantes, meningites, síndrome hemolítica urêmica e sepse. Essas infecções nosocomiais assumem papel de destaque especialmente quando reportadas em grupos suscetíveis como crianças, idosos e portadores de doenças crônico-degenerativas, muitos destes internados em unidades hospitalares em todo o mundo $0^{921222324}$.

No Brasil, estudos revelaram o isolamento de Aeromonas a partir de diferentes nichos como o ecossistema marinho, amostras de solo, alimentos como pescado, mariscos e vegetais e também a partir de infecções em animais domésticos, mamíferos marinhos e animais de sangue frio. Destaca-se também a característica de sazonalidade observada em alguns estudos, os quais revelaram que épocas de temperaturas ambientais elevadas (verão) apresentaram maiores índices de isolamento destes patógenos 512141718 .

A biodiversidade de habitats onde estes patógenos têm sido isolados assume destacado papel para a saúde pública no que se refere à pacientes em Unidades de Terapia Intensiva (UTI). Entre estes pacientes, os neonatos representam importante grupo de risco, pois infecções podem ser favorecidas devido às práticas hospitalares como o uso de cateter endovenoso, intervenções cirúrgicas de várias magnitudes, antibioticoterapia e administração de corticoesteróides. Em nosso meio, a prevenção e controle de sepse seguida de óbito nestes pacientes representam um desafio para as equipes de controle de infecção hospitalar das instituições públicas e privadas ${ }^{91624}$.

A presente investigação, objetivou avaliar a presença de Aeromonas spp e seu perfil de suscetibilidade aos antimicrobianos em amostras de swabs retais de neonatos hospitalizados no município do Rio de Janeiro.

\section{MATERIAL E MÉTODOS}

Foram avaliados 2.323 swabs retais de neonatos com sintomas de diarréia aguda hospitalizados em unidades de terapia intensiva de três hospitais municipais no Rio de Janeiro, no período de 1998 a 2006 . As amostras foram coletadas pelos profissionais de saúde destes hospitais durante a rotina e antes do início da antibioticoterapia.

Após a coleta, as amostras foram transportadas em meio CaryBlair, acondicionadas em recipiente isotérmico contendo gelo reciclável e encaminhadas ao Laboratório de Referência Nacional de Cólera e outras Enteroinfecções Bacterianas, Instituto Oswaldo Cruz, FIOCRUZ, onde as análises microbiológicas foram efetuadas num prazo máximo que não excedeu duas horas.

0 procedimento de isolamento incluiu o enriquecimento direto dos swabs enviados em meio de água peptonada alcalina (APA) acrescida de $1 \%$ de cloreto de sódio $(\mathrm{NaCl})$ e água peptonada alcalina adicionada de $3 \%$ de $\mathrm{NaCl}\left(37^{\circ} \mathrm{C} / 24 \mathrm{~h}\right)$. Em sequiência foi realizada semeadura em meio agar seletivo para PseudomonasAeromonas (GSP) e incubadas a temperatura de $37^{\circ} \mathrm{C}(18-24 \mathrm{~h})$. As colônias suspeitas ( 5 a 10) foram repicadas para meios de triagem (kligler iron agar e lysine iron agar), agar nutriente acrescido de 1\% de cloreto de sódio $(\mathrm{NaCl})$ e, em seguida realizada caracterização bioquímica, com base nos testes de citocromo-oxidase, resistência ao agente vibriostático 0129 (2,4 diamino, 6,7 diisopropilpteridine), produção de orto-nitrofenil-beta-D galactosidase (ONPG), produção de acetoína em meio Voges-Proskauer, fermentação da glicose, sacarose, arabinose e manose e utilização de aminoácidos (lisina e ornitina descarboxilase e arginina dehidrolase) a fim de obter a identificação conclusiva das cepas isoladas ${ }^{16}$.

Subsequiente ao isolamento as cepas de Aeromonas isoladas foram submetidas ao teste de suscetibilidade aos agentes antimicrobianos utilizando os seguintes fármacos: cloranfenicol 30mcg (CHL), cefoxitina 30mcg (FOX), ceftriaxona 30mcg (CRO), nitrofurantoína $300 \mathrm{mcg}$ (NIT), cefalotina $30 \mathrm{mcg}$ (CEP), ácido nalidíxico 30mcg (NIT), ciprofloxacina $5 \mathrm{mcg}$ (CIP), gentamicina $10 \mathrm{mcg}$ (GEN), imipenem 10mcg (IMP), sulfametoxazoltrimetoprim $23,75 \mathrm{mcg} / 1,25 \mathrm{mcg}$ (SXT) e tetraciclina $30 \mathrm{mcg}$ (TCY), através do método de difusão em disco segundo as recomendações do CLSI, $2006^{4}$.

\section{RESULTADOS E DISCUSSÃO}

Em nosso meio, as espécies de Aeromonas que apresentam elevados índices de isolamento em associação à patogenia humana são Aeromonas hydrophila, Aeromonas caviae e Aeromonas veronii biogrupo sobria. Esses microrganismos têm sido isolados de diferentes nichos ecológicos e considerados de importância para a prática médica, pois há relatos de isolamento a partir de amostras clínicas humanas e casos de diarréia infantil ${ }^{716}$.

$\mathrm{Na}$ presente investigação, foram identificadas 8 espécies de Aeromonas isoladas a partir de amostras de neonatos hospitalizados em Unidades de Terapia Intensiva. Entre as espécies isoladas observou-se o isolamento de 56 cepas, assim distribuídas: Aeromonas caviae em 42,8\% dos isolados, seguida deAeromonas media (25\%), Aeromonas veronii biogrupo sobria (10,7\%), Aeromonas hydrophila (9\%), Aeromonas veronii biogrupo veronii (5,3\%), Aeromonas sobria, Aeromonas jandaei e Aeromonas schubertii (1,8\% cada) e uma cepa caracterizada como Aeromonas sp (Tabela 1).

Tabela 1 - Principais espécies de Aeromonas isoladas de neonatos hospitalizados no Rio de Janeiro, no período de 1998 a 2006.

\begin{tabular}{lcc}
\hline Microrganismos & Número & Percentagem \\
\hline Aeromonas caviae & 24 & 42,8 \\
Aeromonas media & 14 & 25,0 \\
Aeromonas veronii biogrupo sobria & 6 & 10,7 \\
Aeromonas hydrophila & 5 & 9,0 \\
Aeromonas veronii biogrupo veronii & 3 & 5,3 \\
Aeromonas schubertii & 1 & 1,8 \\
Aeromonas sobria & 1 & 1,8 \\
Aeromonas jandaei & 1 & 1,8 \\
Aeromonas sp & 1 & 1,8
\end{tabular}

Fonte: Laboratório de Referência Nacional de Cólera e outras Enteroinfecções Bacterianas/Instituto Oswaldo Cruz/Fundação Oswaldo Cruz. 
A maioria das espécies de Aeromonas isoladas tem sido associada à gastrenterite humana incluindo casos denominados diarréia dos viajantes. Aeromonas caviae representou o principal patógeno isolado neste estudo e sua importância está relacionada ao seu elevado potencial de virulência, especialmente em casos de sepse e infecções oportunistas em pacientes imunocomprometidos ${ }^{24}$. É digno de nota ressaltar o isolamento da espécie Aeromonas media, o que não é muito comum a partir de casos clínicos. Paralelamente, a identificação de Aeromonas bydrophila com perfil de resistência aos antimicrobianos representa um dado epidemiológico importante para a saúde pública, corroborando com outros estudos associados à gastrenterite aguda em crianças ${ }^{112122}$.

Embora, o isolamento de algumas espécies de Aeromonas possa ser considerado discreto por sua baixa frequiência, cabe salientar que Aeromonas sobria tem sido isolada como patógeno humano em infecções entéricas. Estudos revelam que a maioria das cepas desta espécie possui uma toxina similar à toxina colérica e cerca de $20 \%$ dos pacientes acometidos pela doença apresentam sintomas de disenteria semelhante às provocadas por espécies de Shigella e cepas de Campylobacter jejuni invasoras ${ }^{215}$.

Por outro lado, Aeromonas schubertii e Aeromonas jandaei assumem importância clínico-epidemiológica associada ao isolamento a partir de infecção de lesões cutâneas subsequientes à exposição à água ou solo contaminado ${ }^{39}$. Esses dados reforçam a importância de alertar aos profissionais de saúde quanto à necessidade de higienização das mãos antes e após manipulação direta ou indireta dos pacientes, bem como da observação das normas gerais de biossegurança hospitalar.

Há relatos de isolamento de cepas de Aeromonas veronii biogrupo sobria potencialmente virulentas a partir de casos de gastrenterite humana, bem como casos de infecção por Aeromonas trota acometendo crianças. Embora, o papel das Aeromonas como agentes causadores de gastrenterite permaneça não totalmente elucidado, estes patógenos tem sido epidemiologicamente associados à ocorrência de diarréia aguda em crianças 2101320

Das 56 cepas de Aeromonas isoladas, um percentual de 26,78\% apresentou perfil de resistência e multirresistência aos antimicrobianos, distribuídas entre as seguintes espécies: Aeromonas caviae, Aeromonas media, Aeromonas veronii biogrupo sobria, Aeromonas veronii biogrupo veronii, Aeromonas hydrophila eAeromonas schubertii. Essas espécies apresentaram diferentes perfis frente ao teste de suscetibilidade antimicrobiana merecendo destaque para a multirresistência em Aeromonas caviae (TCY-CEP-FOX) e Aeromonas veronii biogrupo veronii (SXT-CHL-TCY-NIT). Contudo, perfis de resistência observados para Aeromonas schubertii, Aeromonas caviae e Aeromonas veronii biogrupo veronii para cefalosporinas de terceira geração é um relato de particular interesse clínico (Tabela 2).

Observa-se que a resistência a cefalotina tem sido usada para diferenciar Aeromonas veronii biogrupo veronii de Aeromonas bydrophila. Entretanto, as cepas destas espécies isoladas neste estudo apresentaram sensibilidade frente a este fármaco ${ }^{15}$. Estudos
Tabela 2 - Perfis de Resistência aos Antimicrobianos em Aeromonas spp isoladas de neonatos hospitalizados no Rio de Janeiro, no período de 1998 a 2006.

\begin{tabular}{llc}
\hline $\begin{array}{l}\text { Perfil de Resistência aos } \\
\text { Antimicrobianos }\end{array}$ & Microrganismos & Total \\
\hline FOX & Aeromonas schubertii & 1 \\
& Aeromonas caviae & 1 \\
& Aeromonas veronii biogrupo veronii & 1 \\
\hline SXT & Aeromonas caviae & 1 \\
& Aeromonas media & 2 \\
\hline TCY & Aeromonas veronii biogrupo sobria & 2 \\
\hline TCY-NAL & Aeromonas veronii biogrupo sobria & 1 \\
\hline TCY-CHL & Aeromonas media & 1 \\
\hline TCY-CEP-FOX & Aeromonas caviae & 1 \\
\hline SXT-CEP & Aeromonas caviae & 1 \\
\hline SXT-TCY & Aeromonas caviae & 1 \\
\hline SXT-CHL-TCY-NIT & Aeromonas hydrophila & 1 \\
\hline
\end{tabular}

FOX: ceftriaxona, SXT: sulfametoxazol-trimetoprim, TCY: tetraciclina, NAL: ácido nalidíxico, CHL: cloranfenicol, CEP: cefalotina, NIT: nitrofurantoína.

Fonte: Laboratório de Referência Nacional de Cólera e outras Enteroinfecções Bacterianas/Instituto Oswaldo Cruz/Fundação Oswaldo Cruz.

conduzidos na Índia revelaram cepas de Aeromonas resistentes ao ácido nalidíxico, cefalotina dentre outros antimicrobianos. Com relação ao ácido nalidíxico apenas uma cepa de Aeromonas veronii biogrupo sobria apresentou perfil de resistência para esta droga em associação à tetraciclina ${ }^{21}$.

A circulação de espécies de Aeromonas multiresistentes entre neonatos hospitalizados em unidades de terapia intensiva pode potencializar os riscos de disseminação dos genes de resistência através de plasmídeos ou transposons, o que implica na necessidade de contínuo monitoramento da resistência antimicrobiana, especialmente no ambiente hospitalar .

Portanto, ao considerarmos as implicações clínicoepidemiológicas das espécies de Aeromonas isoladas no presente estudo, bem como a identificação de perfis de resistência e multirresistência aos principais antimicrobianos de uso corrente na prática médico-hospitalar, observamos a necessidade urgente de alertar e esclarecer a verdadeira importância destes patógenos para a Saúde Pública. Esse conhecimento poderá auxiliar positivamente no estabelecimento de diretrizes de controle preventivo de infecções hospitalares em unidades de terapia intensiva neonatal das redes pública e privada no Rio de Janeiro e em todo o território nacional.

\section{REFERÊNCIAS}

1. Abbott SL, Cheung WKW, Janda JM. The genus Aeromonas: biochemical characteristics, atypical reactions, and phenotypic identification schemes. Journal of Clinical Microbiology 41: 2348-2357, 2003.

2. Albert MJ, Ansaruzzaman M, Talukder KA, Chopra AK, Kuhn I, Rahman M, Faruque ASG, Islam MS, Sack RB, Mollby R. Prevalence of Enterotoxin Genes in Aeromonas spp. isolated from children with diarrhea, healthy controls, and the environment. Journal of Clinical Microbiology 38: 785-3790, 2000.

3. Carnahan AM, Maril MA, Fanning GR, Pass MA, Joseph SW. Characterization of Aeromonas schubertii strains recently isolated from traumatic wound infections. Journal of Clinical Microbiology 27: 1826-1830, 1989. 
4. Clinical and Laboratories Standards Institute. Performance standards for antimicrobial susceptibility testing fifteenth informational supplement M-100-S15. Wayne (PA): inical and Laboratories Standards Institute, 2006.

5. Evangelista NSB, Vieira RHSF, Carvalho FCT, Torres RCO, Sant'anna ES, Rodrigues DP, Reis CMF. Aeromonas spp. isolated from oysters (Crassostrea rhizophorae) from a natural oyster bed, Ceará, Brazil. Revista do Instituto de Medicina Tropical de São Paulo 48: 129-133, 2006.

6. Food and Drug Administration (FDA). Bacteriologycal Analytical Manual. $7^{\text {th }}$ edition, 1992

7. Hay J, Seak DV. Ornamental fish: look but not touch. Journal of Royal Society Medicine 89: 359, 1996

8. Hirsch D, Pereira Jr DJ, Logato PVR, Piccoli RH, Figueiredo HCP. Identificação e resistência a antimicrobianos de espécies de Aeromonas móveis e isoladas de peixes e ambientes aquáticos. Ciência Agrotécnica 30: 1211-1217, 2006.

9. Hofer E, Reis CMF, Theophilo GND, Cavalcanti VO, Lima NV, Henriques MFCM. Envolvimento deAeromonas em surto de doença diarréica aguda em São Bento do Una, Pernambuco. Revista da Sociedade Brasileira de Medicina Tropical 39:217-219, 2006.

10. Joseph SW, Carnahan AM, Brayton PR, Fanning GR, Almazan R, Drabick C, Trudo $\mathrm{Jr}$ EW, Colwell RR. Aeromonas jandaei and Aeromonas veronii dual infection of a human wound following aquatic exposure. Journal of Clinical Microbiology 29: 565-569, 1991

11. Juan HJ, Tang RB, Wu TC, Yu KW. Isolation of Aeromonas hydrophila in children with diarrhea. Journal of Microbiology and Immunology Infection 33: 115-117, 2000.

12. Koelle K, Pascual M, Yunus M. Pathogen adaptation to seasonal forcing and climate change. Proceedings Biology Science 272: 971-977, 2005.

13. Leblanc M, Delage G, Rousseau E, Bonnin ACB. Prévalence d'Aeromonas spp. Dans les gastro-entérites de l'enfant. Canadian Medical Association Journal 138: 714-717, 1988.

14. Moro EM, Weiss RDN, Friedrich RSC, Vargas AC, Weiss LHN, Nunes MP.Aeromonas bydrophila isolated from cases of bovine seminal vesiculites in South Brazil. Journal of Veterinary Diagnostic Investigation 11: 189-191, 1999.
15. Moyer NP. Clinical significance of Aeromonas species isolated from patients with diarrhea. Journal of Clinical Microbiology 25: 2044-2048, 1987.

16. Ong KR, Sordillo E, Frankel E. Unusual case of Aeromonas bydrophila endocarditis. Journal of Clinical of Microbiology 29: 1056-1057, 1991.

17. Pereira CS. A cultura de mexilhões na Baía de Guanabara e suas implicações para a Saúde Pública - Contexto Político-Social e Microbiológico. Tese de Doutorado, Escola Nacional de Saúde Pública Sérgio Arouca, RJ, 2003.

18. Pereira CS, Amorim SD, Santos AFM, Siciliano S, Moreno IMB, Ott PH, Rodrigues DP. Vibrio spp. isolados de mamíferos marinhos capturados na região litorânea do sudeste ao sul do Brasil. Pesquisa Veterinária Brasileira 27: 81-83, 2007.

19. Pereira CS, Possas CA, Viana CM, Rodrigues DP. Aeromonas spp. e Plesiomonas shigelloides isoladas a partir de mexilhões (Perna perna) in natura e précozidos no Rio de Janeiro, RJ. Ciência e Tecnologia de Alimentos 24: 562-566, 2004 .

20. Reina J, Lopez A. Gastroenteritis caused by Aeromonas trota in a child. Journal of Clinical Pathology 49: 173-175, 1996.

21. Sinha S, Shimada T, Ramamurthy T, Battacharya SK, Yamasaki S, Takeda Y, Nair GB. Prevalence, serotype distribution, antibiotic susceptibility and genetic profiles of mesophilic Aeromonas species isolated from hospitalized diarrhoeal cases in Kolkata, India. Journal of Medical Microbiology 53: 527-534, 2004

22. Subashkumar R, Thayumanavan T, Vivekanandhan G, Lakshmanaperumalsamy P. Occurrence of Aeromonas hydrophila in acute gasteroenteritis among children. Indian Journal of Medical Research 123: 61-66, 2006.

23. Tena D, González-Praetorius A, Simeno C, Pérez-Pomata MT, Bisquert J. Extraintestinal infection due to Aeromonas spp.: a review of 38 cases. Enfermedades Infecciosas y Microbiología Clínica 25: 235-241, 2004.

24. Wang G, Tyler KD, Munro CK, Johnson WM. Characterization of cytotoxic, hemolytic Aeromonas caviae clinical isolates and their identification by determining presence of a unique hemolysin gene. Journal of Clinical Microbiology 34: 3203-3205, 1996. 